\title{
Removing Barriers to Access Starts with a Phone Call: Impact of Pre-Visit Coordination of Care in Efficiency of Therapy and Patient and Provider Satisfaction
}

\author{
Andrew J. Sinnamon, MD, MSCE, Pamela J. Hodul, MD, and Jose M. Pimiento, MD \\ Department of Gastrointestinal Oncology, Moffitt Cancer Center and Research Institute, Tampa, FL
}

It is well known to surgeons, physicians, and in general to oncologic providers, that one of the most important moments for patients is the first clinic appointment for consultation following a new cancer diagnosis. The lack of centralized health care records and the challenges in communication between health care providers makes this visit ripe for several potential issues. Additionally, the agenda for the first clinic appointment is often wide ranging-from discussing the implications of a new diagnosis and addressing the patient's anxiety and fears, to reviewing the results of diagnostic tests and determining which studies are still needed, and finally to outlining potential treatment plans and their implications to the patient and their family. Addressing all of these issues in one sitting is frequently challenging. This is especially the case in specialty cancer centers and tertiary and quaternary referral centers where cases are often complex in nature. Independent of this, complete staging is a requisite before treatment planning can begin and patients rarely arrive at their first appointment with a complete workup as indicated per national guidelines. As a result of this, there is inefficient use of the initial clinic appointment; we often find ourselves describing potential steps that might be taken depending on the results of future workup, rather than outlining the actual plan of care based on results of workup. In an ideal world, this workup would be undertaken in parallel and completed while scheduling the first

(C) Society of Surgical Oncology 2021

First Received: 12 November 2021

Accepted: 17 November 2021;

Published Online: 1 December 2021

J. M. Pimiento, MD

e-mail: Jose.pimiento@moffitt.org consultation. In the absence of this, novel care paradigms may be developed to reduce inefficiency, improve patient experience, and expedite time to initiation of care.

In this retrospective analysis by Vos, Strong, and colleagues, the surgical oncology team at an NCCN designated cancer center, Memorial Sloan Kettering Cancer Center (MSKCC), examine their experience with one such endeavor - the implementation of a new standard of care in their institution, the Enhanced Patient Clinical Streamlining Process (EPACS). ${ }^{1}$ The EPACS intervention consisted of early review of patient documentation followed by a phone call to the patient by an APP "to achieve familiarity, provide simple education, answer questions, and obtain medical history" in preparation for the first clinic consultation, with early ordering of workup as appropriate. EPACS became the standard of care at their institution following encouraging results of a pilot implementation period. The authors compare this experience with a control group of patients who were not part of the EPACS intervention.

The results presented illustrate a relatively low-cost intervention in terms of administrative workload, with a return-on-investment of meaningful outcomes. The majority of EPACS calls took 10-30 min (10-20 min 48\%, 20-30 min 24\%). The interaction as a whole required a median of $38 \mathrm{~min}$ to prepare for, perform the call, and coordinate care afterward. As a result, imaging testing and diagnostic procedures were ordered before the first clinic appointment more commonly in the EPACS group when compared with patients who did not receive an EPACS call ( $34 \%$ vs. $16 \%$ ), making more efficient use of the pre-clinic waiting period. Furthermore, the cancellation rate of the first appointment was significantly reduced (4.6\% vs. $12 \%$ ), which is likely a reflection of improved patient engagement and satisfaction of care received by the patient 
considering that their cancellations are typically due to receiving care at another competing facility. Importantly, the authors found that this early communication translated to a shorter time to initiate oncologic treatment (median 17 days vs. $19, p=0.086$ ).

Perhaps the most compelling results from this study appear to be from the perspective of the patient. Twentynine of $31(94 \%)$ patients surveyed following the intervention felt that the phone call reduced their anxiety, and an overwhelming majority of these (26/29) reported that their anxiety was reduced by 8 or more points on a 10-point scale. Further, patients felt largely better prepared for the first visit (27/29 by 8 points or more). As we all know, patients unfortunately do not retain much of what is discussed in the initial consultation appointment as they are still grappling with the new information of a cancer diagnosis. While impossible to quantify in this study, it is reasonable to hypothesize that this may be improved to some degree when patients are functioning at a dramatically reduced anxiety level. These results were achieved at a relatively modest cost, as only 3 of 17 surveyed APPs felt an increased workload burden while, conversely, over half $(56 \%)$ of surveyed care providers felt overall workload was decreased. Overall, the majority of surgeons and APPs felt that the productivity of the clinic appointment was improved with the use of EPACS (27/33, 82\%).

The experience at MSKCC is consistent with other endeavors to improve streamlining of patient care in oncology. Often, cancer patients are otherwise healthy with limited prior exposure to the healthcare system and are suddenly faced with a life-changing diagnosis. They are eager to rapidly move through the necessary steps to be ready for the initiation of therapy. The use of oncology nurse navigators is a well-established strategy to facilitate this process with clearly documented benefits. Nurse navigator support in the short term following new early cancer diagnoses has been shown to improve patient-reported experience and may reduce cost. ${ }^{2,3}$ Communication with a nurse navigator may additionally provide value to the initial appointment when performed beforehand. ${ }^{4}$

Our institution has undertaken similar strategies to improve the value of the initial clinic appointment visit and reduce inefficiencies in the initiation of cancer care. We similarly have a practice of APP-patient communication prior to initial clinic consultation with the goal of answering questions, obtaining basic medical history, and establishing goals for the first clinic appointment. We have generally found favorable outcomes with this approach (data not published). As an additional intervention, a novel care paradigm has been introduced within our hepatobiliary division wherein new patients are reviewed and discussed in a multidisciplinary tumor board prior to their first clinic appointment, with the goal of having a more meaningful first clinic visit. In a pre- and post-intervention analysis, this was shown to be associated with significantly reduced time to initiation of therapy. ${ }^{5}$ This practice model has since been adopted by other divisions within our cancer center.

As acknowledged by the authors, the rate of EPACS interventions at MSKCC was relatively low (14\%) despite this being advocated as a new institutional standard of care. This was felt to be related to barriers to adoption of this practice. Absence of some level of expertise in the patient's cancer diagnosis may result in inaccurate information and or an inefficient connection. More formal training may help reduce this low adoption rate. Furthermore, designated time for pre-clinic communication allocated to the schedule may also increase this rate. Given a more efficient subsequent clinic experience, this would have a positive return on investment of time and resources. As proposed by the authors, it should be emphasized that pre-visit communication would be an excellent opportunity to minimize gaps in healthcare disparities. If pre-appointment communication is not possible in all patients due to resource and time constraints, those at risk for delays in care for socioeconomic reasons should be a focus for these efforts. In particular, there should be a focus toward approaching the patient in their language of preference to reduce inherent socioeconomic bias that would otherwise result.

In order to support the EPACS system, several new resources required development at MSKCC, such as shared patient documentation prior to the creation of the electronic medical record entry and computer-automated reports listing new patients scheduled for visits. Given systematic differences between institutions, the strategy outlined by Vos et al. may not apply to other hospitals and centers. Providers would likely need to individualize this practice to tailor to the idiosyncrasies of their particular institution. Given this study and others' findings of favorable results, particularly with regard to patient-reported satisfaction and reduction in anxiety, these are noble efforts that should be encouraged and emulated as we strive to improve care for our oncology patients.

\section{REFERENCES}

1. Vos EL, Cho JS, Schmeltz J, Teri N, Law EB, Paisley K, et al. Enhanced Patient Clinical Streamlining (EPACS): quality initiative to improve healthcare for new surgical outpatient visits. Ann Surg Oncol.. 2021. https://doi.org/10.1245/s10434-021-11126-3.

2. Wagner EH, Ludman EJ, Aiello Bowles EJ, Penfold R, Reid RJ, Rutter CM, et al. Nurse navigators in early cancer care: a randomized, controlled trial. J Clin Oncol. 2014 Jan 1;32(1):12-8.

3. Loiselle CG, Attieh S, Cook E, Tardif L, Allard M, Rousseau C, et al. The nurse pivot-navigator associated with more positive cancer care experiences and higher patient satisfaction. Can Oncol Nurs J. 2020 Jan 1;30(1):48-53.

4. Wang T, Huilgol YS, Black J, D'Andrea C, James J, Northrop A, et al. Pre-Appointment Nurse Navigation: Patient-Centered 
Findings From a Survey of Patients With Breast Cancer. Clin J Oncol Nurs. 2021 Oct 1;25(5):E57-E62.

5. Ehab J, Powers B, Kim R, Haider M, Utuama O, Chin A, et al. Integrating a Disease-Focused Tumor Board as a Delivery-of-Care Model to Expedite Treatment Initiation for Patients With Liver Malignancies. Ann Surg Oncol. 2021 Oct 20. https://doi.org/10. 1245/s10434-021-10909-y. Epub ahead of print.
Publisher's Note Springer Nature remains neutral with regard to jurisdictional claims in published maps and institutional affiliations. 